level perspective in the analysis. All costs and benefits were discounted to the beginning of the exposure reduction period. Various sensitivity analyses were undertaken with key parameters.

Findings suggest the net benefit is positive from 2055 onward for both reduction approaches, i.e., there is a positive return on investment at the societal level. The largest component of benefits is from health-related quality of life gains, with productivity/output gains accounting for the second largest component. Healthcare savings is the smallest component, primarily because cancer cases incur relatively modest health costs due to their short life-expectancy following diagnosis.

Promoting the use of personal protective equipment and engineering controls across the construction sector can substantially reduce exposures to silica dust and give rise to net benefits at the societal level in terms of improvements in health-related quality of life, increased productivity/output, and reduce healthcare costs.

\section{O3C.3 LONGITUDINAL ASSESSMENT OF SMOKING CESSATION AND MORTALITY FROM ALL-CAUSE AND ALL-CANCER AMONG SILICOTICS IN HONG KONG, 1981-2014}

${ }^{1}$ Lap Ah Tse* ${ }^{2}$ Chi Kuen Chan, ${ }^{1}$ Ignatius Tak-sun Yu, ${ }^{3}$ Chi Chiu Leung. ${ }^{1} J C$ School of Public Health and Primary Care, The Chinese University of Hong Kong, Hong Kong SAR, China; ${ }^{2}$ Pneumoconiosis Clinic, Tuberculosis and Chest Service, Department of Health, Hong Kong SAR, China; ${ }^{3}$ Stanley Ho Centre for Emerging Infectious Diseases, The Chinese University of Hong Hong, Hong Kong SAR, China

\subsection{6/OEM-2019-EPI.69}

Background Occupational epidemiological studies among silicotics showed that long-term smoking cessation had lowered lung cancer risk by over $50 \%$, but the beneficial effect for reducing risks of other diseases remains unknown. We aimed to evaluate the impact of smoking cessation on the mortality from all-cause and all-cancer using a large historical cohort of 3185 Chinese silicotics since 1981 and followed-up till 2014.

Methods Each silicotic's baseline information was collected including socio-demographics, occupational history and medical history. Smoking habits were assessed at the baseline and reassessed during the follow-up. Multiple Cox proportional hazards model was performed to evaluate the impact of smoking cessation on all-cause and all-cancer mortality using adjusted hazard ratio (AHR) and 95\% confidence interval $(95 \% \mathrm{CI})$.

Results By the end of 2014, a total of 1942 deaths occurred and 360 silicotics died from cancer. Compared with never smokers, silicotics who were new quitters had $30 \%$ and $65 \%$ higher risk of all-cause of death $[\mathrm{AHR}=1.30,95 \% \mathrm{CI}$ : 1.06-1.58] and all-cancer $(\mathrm{AHR}=1.65,95 \% \mathrm{CI}: 1.04-2.62)$, while persistent quitters had a $52 \%$ and $49 \%$ excess risk of all-cause of death $(\mathrm{AHR}=1.52$, 95\% CI: 1.25-1.84) and allcancer $(\mathrm{AHR}=1.49,95 \% \mathrm{CI}: 0.94-2.36)$, respectively. AHR for all-cause mortality among never quitters was $1.40(95 \%$ CI: 1.14-1.73) while the HR for all-cancer was $2.08(95 \%$ CI: 1.30-3.32). Both all-cause mortality and all-cause mortality decreased sharply after 5 years of smoking cessation and their risks almost equaled to those of the never smokers if the quitters could have kept abstained for more than 20 years.
Conclusions Smoking cessation sharply decreased all-cause and all-cancer mortality among workers with silicosis, and the beneficial effect was prominent for the long-term quitters.

Acknowledgement Pneumoconiosis Compensation Fund Board, Hong Kong

\section{C.4 INCREASES THE RISK OF SARCOIDOSIS BY SILICA EXPOSURE? A CASE-CONTROL STUDY}

${ }^{1}$ Per Vihlborg*, ${ }^{2}$ Pål Graf, ${ }^{1}$ Ing-Liss Bryngelsson. 'Department of Occupational and Environmental Medicine, Faculty of Medicine and Health, Örebro University, Örebro, Sweden; ${ }^{2}$ National Institute of Occupational Health, Oslo, Norway

\subsection{6/OEM-2019-EPI.70}

Introduction Sarcoidosis is an inflammatory disease with unknown etiology that involves the formation of granulomas, mainly in the lungs and/or intrathoracic lymph nodes. Hypothesis about the etiology of sarcoidosis are combination of genetic and environmental factors. Previous studies have linked exposure to silica dust with increased risk of sarcoidosis.

Object A case-control study to investigate the silica exposure among Swedish sarcoidosis cases.

Methods The data was collected from the National non-primary outpatient care register kept by the Swedish National Board of Health and Welfare. All cases between the age of 20 and 65 with the diagnosis Sarkoidos-D86 according to the International Classification of Diseases, 10th Edition (ICD-10) was included in the study (11 772 cases). The information was matched towards the register for cause of death and the register for emigration.

For each case two controls were included matched for age, sex, was resident in the same county, should not be first degree relatives to cases and not have been diagnosed with the investigated disease using the Swedish Central Bureau of Statistics (SCB) multigeneration register. Cases and controls was matched against SCBs occupational registry for work profession. The levels of silica dust exposure were estimated using NOCCA-JEM (Nordic Occupational Cancer study jobexposure matrix) a modified version of the Finnish Information System on Occupational Exposure job-exposure matrix (FINJEM) which is a well-established method of estimating exposure.

Result Cases of sarcoidosis have an increased exposure to silica before diagnosis $(1.19$; 95\% CI 1.1 to 1.30$)$.

Conclusion The increased exposure to silica among sarcoidosis cases suggest that silica can be an environmental factor that contribute to development of sarcoidosis.

\section{C.5 REDUCED SERUM CLARA CELL PROTEIN (CC16) AS AN EARLY PULMONARY INJURY MARKER FOR FINE PARTICULATE MATTER EXPOSURE IN OCCUPATIONAL POPULATION}

Huawei Duan*, Yanhua Wang, Zhenjie Wang, Ting Wang. National Institute of Occupational Health and Poison Control, Chinese Center for Disease Control and Prevention, Beijing, China

\subsection{6/OEM-2019-EPI.71}

Background Particulate matter is the key component of air pollutants, mainly produced by emissions of coal-fired plants and road traffic. Exposure to fine particulate matter $\left(\mathrm{PM}_{2.5}\right)$ pollution is associated with increased morbidity and mortality 